\title{
The limbic system and the localisation of herpes simplex encephalitis
}

\author{
ANTONIO R DAMASIO, ${ }^{*}$ GARY W VAN HOESEN* $\dagger$ \\ From the Departments of Neurology* and Anatomy†, University of Iowa College of Medicine, Iowa City, \\ Iowa, USA
}

SUMMARY The selective destruction of temporal and frontal lobe structures by herpes simplex encephalitis has been explained as a consequence of the proximity of those regions to the point of entry of the virus in the encephalon, through olfactory pathways or meningeal branches of the trigeminal nerves. An alternative hypothesis is presented: that the encephalitis is due to a special affinity of the herpes simplex Type 1 virus for the limbic cortices, that is, that distinctive neuroanatomical, neurochemical and neuroimmunological properties of those cortices permit the virus to manifest its destructive behaviour, regardless of the route of entry to the CNS, possibly during altered immunological states. The study of the neurochemical and neuroimmunological properties of the limbic cortices may be a useful approach to the enigma of why and when herpes simplex Type 1 causes encephalitis.

Despite some advances in antiviral therapy, herpes simplex encephalitis is still a devastating and often fatal disease. ${ }^{1}$ Patients who survive do so with major defects of memory and affect, often incompatible with personal and social rehabilitation. The agent of the encephalitis, the herpes simplex virus Type 1 , resides latent, possibly for many years, in the trigeminal ganglia of its victims. ${ }^{2-4}$ But the reason why the virus abandons its dormant state to invade and destroy the central nervous system remains a mystery. It is also not known why the virus selectively damages some neural structures of the temporal and frontal lobes and spares most other cortices, gray matter nuclear masses and related white matter. Considering that infection with herpes simplex virus Type 1 is highly prevalent in humans, it is important to discover (a) why the virus turns from relatively benign tenant to malignant invader, (b) the route

\footnotetext{
Address for reprint requests: Antonio $\mathbf{R}$ Damasio, $\mathrm{MD} \mathrm{PhD}$, Department of Neurology. University of Iowa Hospitals \& Clinics, Iowa City, IA 52242, USA.
}

Received 8 June 1984 and in revised form 6 September 1984. Accepted 18 September 1984

This work was supported by NINCDS Grant PO1 NS 19632-01. taken to invade the central nervous system, and (c) the reasons why a specific set of neural structures is consistently chosen as target. In this article we address the latter two issues.

The first explanation proposed for the unique localisation of herpes simplex encephalitis invoked intranasal inoculation and spread along the olfactory pathways into the nearby temporal and frontal cortices. ${ }^{5}$ More recently, Davis and Johnson advanced another explanation. ${ }^{6}$ They postulated that from its latent site in the trigeminal ganglia, the virus would travel through the tentorial branches of the trigeminal nerve in the direction of the dura mater. Because the tentorial nerves supply the dura mater in both the middle and anterior fossae, in and about the areas that the mesial and polar temporal lobes and the inferior surface of the frontal lobes directly overlie, ${ }^{7}$ the virus would then travel in cell to cell contact across the meninges and into the subjacent cortices.

Both explanations are plausible but they do not acknowledge (a) that the structures damaged by herpes simplex encephalitis are part of an anatomical and functional neural unit, the limbic system; (b) that the boundaries of that anatomical system are respected, often faithfully, by the destructive process; (c) that if the location of damage would be due 
solely to proximity to the area of entry, then there would be no explanation for why the process rarely trespasses into other territories; (d) that although meningeal branches of the ophthalmic divisions of the trigeminal nerve do innervate dura mater in regions directly across from limbic cortices, they also contribute innervation to other areas of the dura in the middle and anterior fossae,$^{78}$ the overlying cortex of which is generally not involved.

\section{Anatomical and pathological features of herpes encephalitis}

Herpes encephalitis causes selective damage to the gray matter of the entire limbic system, for example the allocortices and the mesocortices (periallocortex and proisocortex). It severely involves hippocampal formation, including the subicular cortices (allocortical structures) and the amygdala, as well as the parahippocampal gyrus; the perirhinal cortex; the agranular insular cortex; the posterior orbitofrontal cortex; the paraolfactory gyrus of Broca; and the anterior cingulate cortex, structures that together constitute the mesocortices. Cytoarchitecturally, the
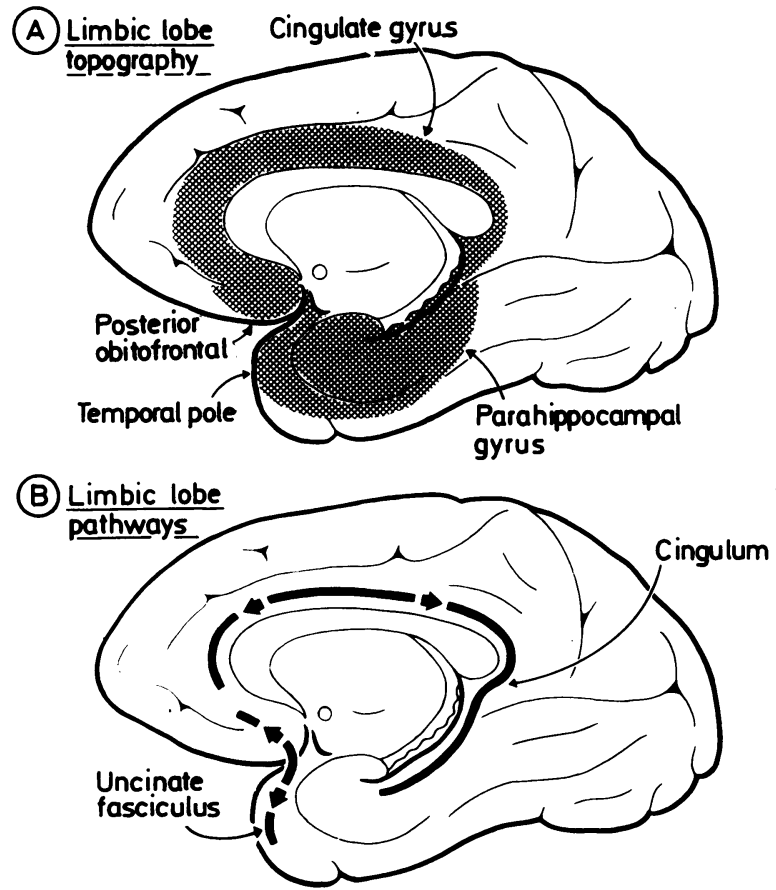

Fig 1 Diagrams of the medial surface of a higher primate brain, depicting: $A$. the topography of the meso and allocortices that constitute the limbic lobe; $B$. two major pathways that interconnect various parts of the limbic lobe; $C$. the topography of allocortical subicular projections to the limbic lobe; $D$. the topography of amygdaloid projections to the limbic lobe. Note how the distribution of these projections coincides with the areas destroyed by herpes simplex encephalitis.

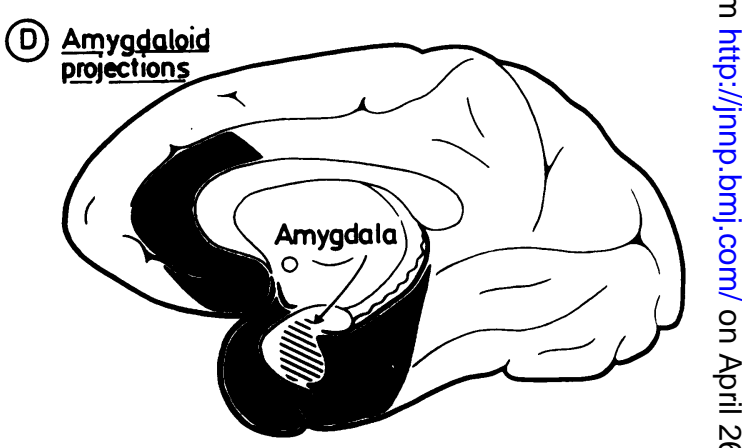

mesocortices are an intermediate structure, less elaborate than the 6-layered isocortex (also known as neocortex) which forms most of the telencephalon but more complex than the allocortices which constitute the hippocampal formation and the superficial parts of the amygdala." Both allocortices and mesocortices are relatively less myelinated and contain neuronal types that are unique as well as microvascular patterns that are unlike those of other cortices. ${ }^{1011}$ Moreover, they contain a high concentration of opiate receptors, ${ }^{12}$ abundant quantities of trace metals, high concentrations of indoleamines, catecholamines, cholinergic enzymes and, in general, less oxidative enzymes than the isocortices. ${ }^{13}$ fact that the mesocortices are powerfully interconnected via large association bundles such as the cingulum and the uncinate fasciculus ${ }^{14}$ and that they are also connected with the subicular allocortices ${ }^{15}$ and with the subcortical amygdaloid nuclei ${ }^{1617}$ (fig 1). The encephalitis consistently involves nearly the totality of the limbic mesocortices as well as the amygdala and hippocampal formation. Only the $\stackrel{p}{\infty}$ posterior cingulate cortex, which is cytoarchitecturally different from the anterior, may escape its pil-

(C) Subicular projections

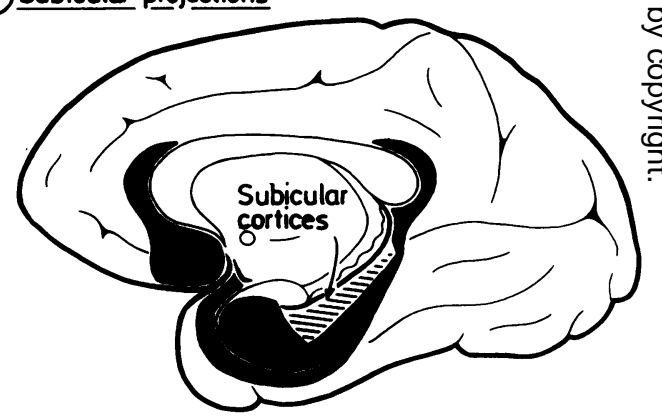
Finally, but perhaps of no lesser importance, is the 
lage. (A note should be made here of the fact that the anterior cingulate gyrus, Brodmann's area 24 , although seldom mentioned as a damaged zone in textbook descriptions, was destroyed in nearly all published cases we reviewed and in all necropsy cases personally observed; the concentration of viral antigen in the anterior cingulate was extremely high, of the same magnitude as that found in other limbic cortices. ${ }^{18}$ ) Furthermore, the boundary between the limbic cortices damaged by herpes simplex encephalitis and the nonlimbic cortices in the vicinity, is remarkably sharp, especially in the cingulate gyrus and in the orbito-frontal region. For instance, necrosis of the cingulate is halted at the cingulate sulcus where the cytoarchitecture changes to give way to the supplementary motor area. In the horizontal plane, necrosis stops at the boundary between Brodmann's area 24 and 23 (the anterior and posterior cingulate regions, respectively). The nearby genu of the corpus callosum is commonly spared. This sharpness of boundaries is often less marked in the temporal lobe component of the lesions where necrosis, haemorrhage and ischaemia caused by the encephalitis affect a wider group of cortical and subcortical structures; the destruction can be massive, with ensuing collapse of regions that extend beyond the frontier of the limbic system. In other words, although it is true that both neurons and glia are involved in the process of herpes encephalitis, we doubt that it would be possible to spare glia selectively considering the type of pathological processes set in motion by the virus. On the other hand, at least in adults, the encephalitis does prefer a specific type and site of neural structure-the oldest cortex in phylogenetic evolution and one that is neuroanatomically and neurochemically distinctive-while it refrains from destroying neural structures that do not belong to that particular type, namely all of the isocortex. The fact that herpes simplex encephalitis in infants fails to respect these cytoarchitectonic boundaries further emphasises the point that, in adults, the response of limbic cortices to this agent is especially distinctive. It is possible, for instance, that the immunologic properties of limbic and non-limbic neurons are different in the adult. The recent description of a monoclonal antibody for membrane proteins which specifically labels cells in the limbic cortices but leaves nonlimbic elements unlabelled, is of special importance. ${ }^{19}$

\section{Hypothesis}

Our hypothesis can be stated as follows: The initial mode of transport of the virus would be in a retro- grade direction from cutaneous receptors to its latent site in the trigeminal ganglion. From there, by means of transganglionic transport across the ganglion cell soma, the virus would travel anterogradely in the central root in the direction of the brainstem and of the trigeminal nuclei. The virus would then spread transneuronally to limbic related nuclei, such as the raphe and locus coeruleus. Those nuclei have well known and widespread ascending projections to mesencephalic, diencephalic and telencephalic areas. Utilising these neural channels the virus would be distributed almost everywhere in the cerebral cortices but only in the limbic cortices of the adult would it find the proper terrain to multiply and destroy nervous tissue, possibly in circumstances of disturbed immunity. The elaborate intrinsic connectivity of the limbic cortices would augment the "seeding" of the virus within that system, again through exclusively normal neural connections.

The evidence in favour of this hypothesis is as follows: (1) in both bilateral and unilateral cases of adult herpes encephalitis, the lesions almost invariably fall, in a monotonous pattern, in the structures of the limbic system reviewed above; (2) damage in these structures is systematic, that is, it leaves no anatomical "gaps" and generally respects cytoarchitectonic boundaries with non-limbic areas; invasion either through peripheral branches of the trigeminal nerves or through the olfactory nerves, would be likely to cause patchier involvement, with islands of spared tissue, and it is difficult to accept that it might respect cytoarchitechtonic frontiers; (3) Esiri's immunohistological study of the distribution of viral antigen in the brains of herpes encephalitis victims shows that the limbic cortices are the sites where viral antigen is concentrated; ${ }^{18}$ the infection is heaviest in the granule cells of the dentate gyrus of the hippocampal formation; it is noteworthy that the locus coeruleus and raphe nuclei project strongly to the hilum of the dentate gyrus and thus their terminals are immediately adjacent to the granule cells that together form a hilum; ${ }^{2021}(4)$ the mapping of viral antigen in brains with herpes encephalitis studied at different stages during the first three weeks post-onset of the disease, suggests a "wave" of infection spreading rapidly from limbic structure to limbic structure; the subacute progression of "limbic" symptomatology could be explained by an accrual of limbic involvement due to this mode of anatomical spread; all along the neural chain the affinity of the virus for certain types of neurons would be the determinant factor, (for example, viral antigen has been found throughout the telencephalon, including the isocortical motor strip, yet necrosis of that type of cortex is rarely reported at necropsy nor are signs related to its dysfunction part of 
the clinical picture of herpes encephalitis); (5) evidence from experimental studies in animals by Kristensson and associates, ${ }^{22}$ suggests that the virus may travel the natural, CNS-bound route of the trigeminal nerve, and that it can infect the main sensory and spinal trigeminal nuclei and even continue along that neural system, transneuronally, toward the reticular formation, the raphe nuclei, the locus coeruleus and sensory thalamus.

A note should be made here of the fact that the olfactory bulb receives input from some brainstem areas that do not receive olfactory bulb projections themselves. Especially remarkable are the raphe and locus projections, which explain the abundant serotoninergic and noradrenergic terminals in the bulb. ${ }^{23}$ This means that the olfactory bulb could be infected via a trigeminal mode of entry, instead of directly, which might account for the inconsistent viral infection noted in the bulb. ${ }^{6}$

Although arguments for a trigeminal route of entry appear, at the moment, more persuasive than arguments for an olfactory route, the issue is far from settled. The bulb might be a conduit rather than a target, in much the same manner of the trigeminal ganglion. The ganglion is only rarely destroyed in herpes encephalitis in spite of its role as reservoir. There is, thus, no reason why the bulb should necessarily be destroyed.

A comment should also be made about Kristensson's studies in animals sacrificed 3 to 4 days after cutaneous inoculation of the virus. The virus had by then travelled to brain stem-nuclei and to the contralateral thalamus but no mention is made of viral presence in cortical limbic system structures or sensory cortex. Assuming that was specifically looked for and not encountered, and leaving aside species differences, we suspect that absence was to be expected considering the time required for intralimbic anatomical spread to take place. In this regard it is important to keep in mind that the concentration of viral antigens found in Esiri's study was dependent upon the time after onset, ${ }^{18}$ and that clinical evidence clearly documents a subacute progression of signs compatible with gradual spread.

In conclusion, we are persuaded that herpes simplex encephalitis is the consequence of a special affinity of the herpes simplex Type 1 virus for the adult limbic cortices (allo, periallo and proisocortices) which permits the virus to manifest its destructive behaviour. That behaviour is not manifested in the phylogenetically newer cortices (isocortex) which the virus can also gain access to but tends not to destroy. The route of entry may be of secondary importance, though it appears that a trigeminal neural route is the most plausible. An altered immunological state may be an adjuvant factor. We suggest that the study of the neurochemical and neuroimmunological properties of the limbic cortices may be a useful approach to the matters of why, when and how herpes simplex Type 1 will strike its host.

\section{References}

' Adams RA, Victor M. Principles of Neurology, 2nd Edition New York. McGraw-Hill Book Company, 1981.

${ }^{2}$ Baringer JR. Herpes simplex virus infection of nervous tissue in animals and man. Prog Med Virol 1975;20: 1-26.

${ }^{3}$ Baringer JR, Swoveland P. Recovery of herpes-simplex virus from human trigeminal ganglions. $N$ Engl J Med 1973;288:648-50.

${ }^{4}$ Bastion FO, Rabson AS, Yee CL, et al. Herpes virus hominis: isolation from human trigeminal ganglion. Science 1972;178:306-7.

5 Johnson RT, Mims CA. Pathogenesis of viral infections of the nervous system. N Engl J Med 1968:278:23-38, 84-92.

- Davis LE, Johnson RT. An explanation for the localization of herpes simplex encephalitis. Ann Neurol 1979;5:2-5.

${ }^{7}$ Penfield W, McNaughton F. Dural headache and innervation of the dura mater. Arch Neurol Psychiat 1940;44:43-75.

${ }^{8}$ Feindel W, Penfield W, McNaughton F. The tentorial nerves and localization of intracranial pain in man Neurology (Minneap) 1960;10:555-63.

${ }^{9}$ Filimonoff IN. A rational subdivision of the cerebral cortex. Arch Neurol Psychiat 1974;58:296-310.

${ }^{10}$ Lorente de No R. Studies on the structure of the cerebra cortex. I. The area entorhinalis, J Psychol Neurol 1983;45:381-438.

"Pfeifer RA. Die Angioarchitektonische areale Gliederund der Groshirnrinde. Leipzig Thieme, 1940.

12 LaMotte CC, Snowman A, Pert CB, Synder SH. Opiate receptor binding in the rhesus monkey brain: association with limbic structures. Brain Res 1978;155:374 9.

${ }^{13}$ Friede RL. Topographic Brain Chemistry, New York Academic Press, 1966.

14 Van Hoesen GW, Pandya DN, Butters N. Some connections of the entorhinal (area 28) and perirhinal (area $35)$ cortices of the rhesus monkey. II Frontal lobe afferents. Brain Res 1975;95:25-38.

${ }^{15}$ Krettek JE, Price JL. Projections from the amygdaloid complex to the cerebral cortex and thalamus in the rat and cat. J Comp Neurol 1977;172:678-722.

${ }^{16}$ Rosene DL, Van Hoesen GW. Hippocampal efferents reach widespread areas of cerebral cortex and amygdala in the rhesus monkey. Science 1977;198:315-7.

17 Porrino LJ, Crane AM, Goldman-Rakic PS. Direct and indirect pathways from the amygdala to the frontal lobe in the rhesus monkey. J Comp Neurol 1981; 198: 121-36.

${ }^{18}$ Esiri MM. Herpes simplex encephalitis: An immunohistological study of the distribution of viral antigen within the brain. J Neurol Sci 1982;54:209-26. 
${ }^{19}$ Levitt P. A Monoclonal Antibody to Limbic System Neurons. Science 1984;223:299-301.

${ }^{20}$ Moore RY, Halaris AE. Hippocampal innervation by serotonin neurons of the midbrain raphe in the rat. $J$ Comp Neurol 1975;164:171-84.

${ }^{21}$ Moore RY. Monoamine neurons innervating the hippocampal formation and septum. In: The Hippocampus. KH Pribram, RL Isaacson eds. New York,
Plenum Press, 1975.

${ }^{22}$ Kristensson K, Nennesmo I, Persson L, Lycke E. Neuron to neuron transmission of herpes simplex virus. J Neurol Sci 1982;54:149-56.

${ }^{23}$ Broadwell RD. Society for Neuroscience Symposia III, Aspects of Behavioural Neurobiology, Neurotransmitter pathways in the olfactory system, Washington Society for Neurosciences 1978;131-66. 